\title{
Love in the Time of COVID:
}

\section{Perceived Partner Responsiveness Buffers People from Lower Relationship Quality}

\section{Associated with COVID-Related Stressors}

\author{
Rhonda N. Balzarini ${ }^{1}$, Amy Muise ${ }^{1}$, Giulia Zoppolat ${ }^{2}$, \\ Alyssa Di Bartolomeo ${ }^{1}$, David L. Rodrigues ${ }^{3}$, María Alonso-Ferres ${ }^{4}$, Betul Urganci ${ }^{5}$, Anik \\ Debrot $^{6}$, Nipat Bock Pichayayothin ${ }^{7}$, Christoffer Dharma ${ }^{8}$, Peilian Chi ${ }^{9}$, Johan Karremans ${ }^{10}$, \\ Dominik Schoebi ${ }^{11}$, and Richard B. Slatcher ${ }^{12}$ \\ ${ }^{1}$ Department of Psychology, York University, Toronto, ON, Canada \\ ${ }^{2}$ Department of Experimental and Applied Psychology, Vrije University, Amsterdam, \\ Netherlands \\ ${ }^{3}$ Iscte-Instituto Universitário de Lisboa, CIS_Iscte, Lisboa, Portugal \\ ${ }^{4}$ Department of Social Psychology, University of Granada, Granada, Spain \\ ${ }^{5}$ Department of Human Development, Cornell University, Ithaca, NY, United States
}


${ }^{6}$ Department of Psychology, University of Lausanne, Lausanne, Switzerland

${ }^{7}$ Life Transitions and Psychology of Time Research Group, The Faculty of Psychology, Chulalongkorn University, Bangkok, Thailand

${ }^{8}$ Dalla Lana School of Public Health, University of Toronto, Toronto, Canada ${ }^{9}$ Department of Psychology, University of Macau, Zhuhai, China

${ }^{10}$ Department of Psychology Behavioural Science Institute, Radboud University, Nijmegen, Netherlands

${ }^{11}$ Department of Psychology, Université de Fribourg, Fribourg, Switzerland ${ }^{12}$ Department of Psychology, University of Georgia, Athens, GA, United States

Correspondence concerning this article should be addressed to Rhonda Balzarini, Department of Psychology Main Office, Texas State University, San Marcos, TX 78666. E-mail: rbalzarini@txstate.edu. 


\begin{abstract}
External stressors can erode relationship quality, though little is known about what can mitigate these effects. We examined whether COVID-related stressors were associated with lower relationship quality, and whether perceived partner responsiveness - the extent to which people believe their partner understands, validates, and cares for them-buffers these effects. When people in relationships reported more COVID-related stressors they reported poorer relationship quality at the onset of the pandemic $(N=3,593$ from 57 countries) and over the subsequent three months $(N=1,125)$. At the onset of the pandemic, most associations were buffered by perceived partner responsiveness, such that people who perceived their partners to be low in responsiveness reported poorer relationship quality when they experienced COVIDrelated stressors, but these associations were reduced among people who perceived their partners to be highly responsive. In some cases, these associations were buffered over the ensuing weeks of the pandemic. Keywords: COVID-19; Stress and coping; Financial Strain; Loneliness; Relationship Quality, Relationship Conflict
\end{abstract}




\section{Love in the Time of COVID: Perceived Partner Responsiveness Buffers People from Lower Relationship Quality Associated with COVID-Related Stressors}

In response to the novel coronavirus (COVID-19) pandemic, stay-at-home policies were implemented across the world, causing widespread financial instability (Congressional Research Service, 2020; Gangopadhyaya \& Garrett, 2020; King, 2020) and drastic changes to people’s ability to socially connect with others (Brooks et al., 2020). Indeed, stay-at-home orders meant that many people in relationships were social distancing together with their partner, with both partners often working and caring for children from home (Carlson, Petts, \& Pepin, under review; Kerr, Rasmussen, Fanning, \& Braaten, 2021). Yet, supportive close relationships are among the most robust predictors of health and well-being (e.g., Holt-Lunstad, Smith, \& Layton, 2010), and recent work finds that a high-quality romantic relationship can be an immense resource for couples coping with COVID-19-related stressors (Williamson, 2021). However, maintaining a high-quality romantic relationship during times of heightened stress - such as in the case of the COVID-19 pandemic - is challenging (e.g., Neff \& Karney, 2004). Past research suggests that couples who experience prolonged financial strain, lack of social connection (e.g., feel lonely), and higher levels of stress are at risk for relationship dissatisfaction and discord (Bodenmann, 1997; Conger, Rueter, \& Elder, 1999; Karney, Story, \& Bradbury, 2005). In the current research, we examine whether stressors related to coping with the COVID-19 pandemic (i.e., loneliness, financial strain, and stress) are associated with relationship quality and conflict, and if so, whether perceived partner responsiveness - the extent to which people believe that their partners understand, validate, and care for them (Reis, 2012; 2013; Reis, Clark, \& Holmes, 2004) — can buffer against the lower relationship quality and greater conflict expected to be associated with greater COVID-related stressors. 


\section{COVID-Related Stressors and Relationship Quality}

Stay-at-home orders, which were mandated throughout the world to mitigate the spread of COVID-19 (Koo, Cook, \& Park, 2020; Lewnard \& Lo, 2020), involved drastic changes to people's daily activities, limiting opportunities for people to stay connected with their friends and family. Yet, social connection is a robust predictor of well-being, and people often find extended periods of social deprivation challenging (e.g., Baumeister \& Leary, 1995). While social ties with coworkers and membership in social groups (e.g., church, sports team, club) can foster a sense of belonging and prevent loneliness (Hawkley, Browne, \& Cacioppo, 2005; Johnson \& Mullins, 1989), these opportunities to socially connect were drastically altered amid COVID-19 stay-at-home orders. This is concerning because loneliness and a lack of an extended social network can take a toll on romantic relationships, with research showing consistent links between social disconnection and lower relationship satisfaction and commitment (Flora \& Segrin, 2000; Segrin, Powell, Givertz, \& Brackin, 2003).

In addition, the COVID-19 pandemic led to an economic downturn with spikes in the unemployment rate worldwide. At its peak, the U.S. saw unemployment rates of $13 \%$ (20.5 million people), a rise that is higher than the Great Recession and Great Depression (Faria e Castro, 2020; Kochhar, 2020; Pappas, 2020). With increased unemployment, individuals likely experienced heightened financial strain and stress affiliated with losing a job, which can erode a person's competence and well-being (Ervasti \& Venetoklis, 2010). Financial strain is also associated with poorer relationship quality (Bodenmann, 1997; Conger et al., 1999; Karney et al., 2005) and with increased conflict and hostility toward partners (for a review see Story \& Bradbury, 2004). Yet, given the wide-reaching economic disruptions introduced by the COVID- 
19 pandemic, many couples likely experienced financial strain and uncertainty unlike any other time in their relationship.

Indeed, past work suggests that external stressors to people's relationships, such as loneliness and financial strain (Conger et al., 1999), are associated with negative relationship processes and poorer relationship functioning, a phenomenon referred to as stress spillover (e.g., Bolger, DeLongis, Kessler, \& Wethington, 1989; Buck \& Neff, 2012; Neff \& Karney, 2004;

Randall \& Bodenmann, 2009). As external stress increases, partners engage in more negative and divisive behaviors (Bolger et al., 1989), and have fewer satisfying interactions (Repetti, 1989). When experiencing high levels of stress, people also tend to make more negative attributions about their partner's behavior (Neff \& Karney, 2004), have more negative evaluations of their relationship (Tesser \& Beach, 1998), engage in more negative communication patterns (Williamson, Karney, \& Bradbury, 2013), and report more relational discord (Karney et al., 2005) compared to low stress periods. In fact, longitudinal studies suggest that stress leads to lower relationship quality and greater relationship discord over time (Bodenmann, 1997; Conger et al., 1999; Neff \& Karney, 2017). Given the accumulating evidence that loneliness, financial strain, and stress can negatively impact romantic relationships, we anticipated that those who experienced higher levels of COVID-related stressors would report poorer relationship quality (i.e., less relationship satisfaction and commitment) and greater relationship conflict.

\section{Moderating Role of Perceived Partner Responsiveness}

Despite emerging work suggesting that stress can spillover into relationships and is linked to poorer relationship quality, some couples may be able to maintain relationship quality in the face of stressful experiences. For example, while negative life events, such as cancer (Gritz, Wellisch, Siau, \& Wang, 1990; Manne \& Badr, 2008; Manne, Ostroff, Rini, Fox, 
Goldstein, \& Grana, 2004), the death of a child (Lehman, Lang, Wortman, \& Sorenson, 1989), and experiencing a natural disaster (Cohan \& Cole, 2002), can be detrimental to relationships, some couples emerge with their relationship intact. One factor that might be particularly important to people's abilities to maintain relationship quality and minimize relationship discord in the face of stress is perceived partner responsiveness - the extent to which individuals believe that their romantic partners care about, understand, and validate their thoughts and feelings (Reis et al., 2004; Reis, 2012; Reis \& Clark, 2013). Perceiving a partner as responsive to the self is essential to healthy social functioning (Slatcher \& Selcuk, 2017; Mikulincer \& Shaver, 2013) and the maintenance of close relationships (Reis et al., 2004), influencing the development and maintenance of relationship intimacy (Laurenceau, Barrett, \& Rovine, 2005). People who perceive their partner as responsive tend to be able to better regulate negative emotions and feel more secure in their relationship (Collins \& Feeney, 2000; Slatcher \& Schoebi, 2017; Slatcher \& Selcuk, 2017), as well as more satisfied and committed to maintaining their relationships over time (Birnbaum, Reis, Mizrahi, Kanat-Maymon, Sass, \& Granovski-Milner, 2016; Gable, Reis, Impett, \& Asher, 2004; Reis et al., 2004; Segal \& Fraley, 2016).

Perceived partner responsiveness may be particularly beneficial to couples in times of stress. In relationships, stress is often characterized by greater disconnection and social withdrawal (Repetti, Wang, \& Saxbe, 2009; Schulz, Cowan, Cowan, \& Brennan, 2004), but perceived partner responsiveness may promote better coping and support provision by facilitating experiences of closeness and open communication (Manne et al., 2018), even during times of stress (Repetti, 1989; Williamson et al., 2013). Perceived partner responsiveness tends to make people feel safe to reveal their needs and vulnerabilities to their partners (Clark \& Lemay, 2010; Laurenceau, Barrett, \& Pietromonaco, 1998) and can help promote confidence that 
a partner is willing to provide responsive support (see Reis, Lemay, \& Finkenauer, 2017), which may be protective against the negative consequences of stressors.

Amidst the stressors introduced by the COVID-19 pandemic, a romantic partner may be a key support provider. Indeed, when individuals encounter threats and stressors, the primary coping strategy for most adults is to turn to their partners for safety and protection (Mikulincer \& Shaver, 2007) and having a responsive partner's support during stressful times can help to alleviate distress. When individuals are faced with a stressor, their partner's responsive support eased anxiety (Collins \& Feeney, 2000; Simpson, Rholes, \& Nelligan, 1992) and may help conflict go better and make people feel safer and more equip to deal with challenges (e.g., Reis et al., 2004; Reis \& Clark, 2013). To be sure, a central function of perceived partner responsiveness involves downregulating anxiety and arousal and instilling a sense of security and comfort (Selcuk, Zayas, \& Hazan, 2010). For example, within relationships characterized by responsiveness, partners show a reduced need for defensive reactions to real or potential failure (Caprariello \& Reis, 2011) and an increased likelihood of self-disclosure (Maisel, Gable, \& Strachman, 2008). This is consistent with the ideas proposed in the Stress Buffering Model (Cohen \& Wills, 1985; Slatcher \& Selcuk, 2017), which suggests that the adverse effects of stress can be buffered by the perceived availability of social support from one's partner. According to this model, we would expect that when individuals perceive their partner as highly responsive to their needs it should dampen the effects of stress and help couples maintain satisfying relationships.

\section{The Current Study}

In the current research, we investigate whether COVID-related stressors-like general levels of stress, loneliness, and financial strain — are associated with reports of relationship 
quality and conflict at the onset of the pandemic and over the next three months. Examining this during a global pandemic provides a unique opportunity to understand the relationship processes that help couples, even those who normally might not be exposed to high levels of stress. While we expected that external stressors would be associated with lower relationship quality, we also wanted to understand a potential protective factor for this spillover effect. That is, we tested whether the association between COVID-related stressors and relationship quality would be moderated by people's perceptions of their partner's responsiveness. We predicted that for people who perceived their partner as low in responsiveness, external stressors to be negatively associated with relationship quality. But, for people who perceived their partner as highly responsive, this effect should be attenuated, such that the association between stressors and lower relationship quality would be weaker or null. The hypotheses were pre-registered on the OSF. ${ }^{1}$

\section{Method}

\section{Participants and Procedure}

Participants were drawn from six waves of the Love in the Time of COVID Study — an ongoing longitudinal project examining the effects of the COVID-19 pandemic on how people connect, relate, and cope over the course of the pandemic (Blind for review). Participants were recruited for the study online from social media sites (e.g., Facebook, Instagram, Reddit), by word of mouth, and through our project website. To be eligible, participants had to be at least 18

\footnotetext{
${ }^{1}$ As the emergence of COVID-19 was rapid and sudden, the design and data collection for this study were expedited to capture people's experiences at the onset of the pandemic and when the implementation of subsequent social distancing measures were first being implemented. The hypotheses for this study were pre-registered during data collection, and no data from this study had been analyzed at the time of our pre-registration with the exception of examining the variables to ensure we had sufficient participant responses. Furthermore, we initially pre-registered the hypotheses outlined in this manuscript using only the baseline data (T1 only; a cross-sectional design; see OSF). However, to offer a more comprehensive understanding of the effects of COVID-related stressors on relationship quality and the buffering effects of perceived partner responsiveness, we decided to expand the study's scope to examine these effects over time in a second pre-registration (T1-T6; longitudinal design; see $\underline{\mathrm{OSF}}$ ) that was created prior to our longitudinal analyses.
} 
years of age and pass one of two attention checks embedded in the survey. A pre-registered power analysis indicated that 2,546 participants would be needed to estimate a small interaction effect $(f=.02)$ with $95 \%$ power (power estimated using G-Power 3.1; Erdfelder, Faul, \& Buchner, 1996; Faul, Erdfelder, Buchner, \& Lang, 2009) at one time point (e.g., at Wave 1). A total of 7,696 individuals accessed the online study, of those, 2,522 were removed because they failed more than one of the three attention checks or were removed for insufficient completion of the survey. ${ }^{2}$ This resulted in 5,174 participants. In the current study, given that our research questions are focused on romantic relationship quality, we analyzed data from the subset of participants who reported currently being in a romantic relationship (e.g., excluding single, divorced, or widowed people, as well as those who indicated that their relationship did not fit within one of the categories provided). The current sample consisted of 3,593 individuals in relationships at Wave 1. Participants were from 57 countries (additional country-level details can be found in the Supplemental Materials), were mainly heterosexual $(82.1 \% ; 11.7 \%$ bisexual, 4.5\% gay/lesbian, $1.7 \%$ did not identify with the options provided), and the majority were women $(77.7 \%)$. Participants were in their early 30's on average $(M=32.35$ years old, $S D=$ $12.45)$ and mostly in long-term $(M=8.34$ years, $S D=9.97)$ dating relationships $(34.4 \%$ married, 8.1\% engaged, $57.5 \%$ dating) and were living at the same place and engaging in social isolation with their partner $(83.7 \%$; $9.4 \%$ were not living together; $6.9 \%$ were living together part-time).

Eligible participants completed an online survey (launched March 27, 2020) shortly after a global pandemic was declared (March 11, 2020) and much of the world had issued shelter-in-

\footnotetext{
2 As commonly employed in the literature (e.g., Berinsky, Margolis, \& Sances, 2014; Curran, 2016), this study included attention check questions, wherein we asked participants to select a particular answer choice for that question (e.g., "Please select "Agree a little." This is not a trick question."). We also excluded participants who completed less than $90 \%$ of the survey as statistical analyses are likely to be biased when more than $10 \%$ of data are missing (Bennett, 2001). We made the decision a priori to exclude participants who did not select the instructed value.
} 
place orders. The survey was initially available in English and was translated to 10 different languages (Spanish, Turkish, Thai, Chinese, Dutch, French, German, Indonesian, Italian, and Portuguese) using back-translation procedures to ensure there were no discrepancies across the different versions of the survey (see Colina, Marrone, Ingram, \& Sanchez, 2017; Tyupa, 2011). The survey asked participants about their experience since the onset of the COVID-19 pandemic (see OSF), including their loneliness, financial strain, stress, perceived partner responsiveness, and relationship quality. Participants who completed Wave 1 of the study were invited to participate in follow-up surveys in which they were asked the same questions every two weeks over the course of three months (for up to six waves of data). Given we are interested in within person changes in COVID-related stressors over the course of the pandemic, in the longitudinal analyses, in addition to the criteria above, we include people who completed at least three surveys, resulting in 1,125 people in the longitudinal analyses. Participation in this study was entirely voluntary and the study procedures were approved by the host institutions prior to beginning research.

\section{Measures}

We assessed the constructs of interest using truncated versions of the measures, or the most representative single item from well-validated scales, to keep the survey as brief as possible to reduce fatigue, increase efficiency, and minimize participant attrition over time (Bolger, Davis, \& Rafaeli, 2003). All measures were assessed at background (Wave 1) and in subsequent follow-up surveys. In the current analyses, we use perceived partner responsiveness at baseline, but for the longitudinal analyses, we use measures of COVID-related stressors and relationship quality at each time point. 
Perceived Partner Responsiveness. The responsiveness subscale of the Perceived Responsiveness and Insensitivity Scale (PRI) Scale (three items; Crasta, Rogge, Maniaci, \& Reis, 2021) was used to assess people's perception of their partners responsiveness. Participants rated items (“'My partner... 'really listened to me', 'tried to see where I was coming from', and 'seemed interested in what I was thinking and feeling"') on a 6-point Likert scale (1 = not at all, $6=$ completely). Items were mean aggregated, with higher scores indicating greater perceived partner responsiveness at Wave $1(\alpha=.90 ; M=4.51, S D=1.15)$.

Loneliness. The UCLA Loneliness Scale (two items; Hughes, Waite, Hawkley, \& Cacioppo, 2004) was used to assess subjective loneliness. ${ }^{3}$ Participants rated items ("In the last two weeks, I felt: 'lonely' and 'isolated'”) on a 5-point Likert scale ( 1 = very slightly/not at all, $5=$ extremely). Items were mean aggregated, with higher scores indicating more loneliness at Wave $1(r(3578)=.63, p<.001 ; M=2.48, S D=1.23)$ and in the follow-up surveys $(M=2.22$, $S D=1.15)$

Financial Strain. Financial strain was assessed using a single item that was originally derived from Pearlin and colleagues (1981) and revised by Okechukwu and colleagues (2012). In the current study, we further revised this measure to specifically ask about financial strain caused by COVID-19. Participants rated the item ("To what degree has the recent COVID-19 outbreak negatively impacted your financial situation?") on a 5-point Likert scale $(1=$ not at all, $5=$ extremely), with higher scores indicating greater perceived financial strain over COVID-19 at Wave $1(M=2.41, S D=1.20)$ and in the follow-up surveys $(M=2.19, S D=1.14)$.

Stress. Stress was assessed using items that were adapted from PANAS (two items; Watson \& Clark, 1999). Participants rated items ("In the last two weeks, I felt: 'stressed' and 'distressed"') on a 5-point Likert scale ( $1=$ very slightly/not at all, $5=$ extremely). Items were

\footnotetext{
${ }^{3}$ Note that the effects are the same with the items assessed individually.
} 
mean aggregated, with higher scores indicating more stress at Wave $1(r(3575)=.62, p<.001$; $M=2.97, S D=1.11)$ and in the follow-up surveys $(M=2.86, S D=1.08)$.

Relationship Satisfaction. A single item from the Perceived Relationships Quality Scale (Fletcher, Simpson, \& Thomas, 2000) was used to assess relationship satisfaction ( "How satisfied are you with your partner?"). Possible responses were on a 7-point Likert scale (1 = not at all, $7=$ extremely), with higher scores indicating more satisfaction at the onset of the pandemic $(M=5.90, S D=1.31)$ and in the follow-up surveys $(M=5.88, S D=1.31)$.

Commitment. A single item from the Perceived Relationships Quality Scale (Fletcher et al., 2000) was used to assess commitment ("How committed are you to your partner?"). Possible responses were on a 7 -point Likert scale $(1=$ not at all, $7=$ extremely), with higher scores indicating higher commitment at Wave $1(M=6.34, S D=1.13)$ and in the followup surveys $(M=6.42, S D=1.07)$.

Conflict. A single item was used to assess conflict ( "Think about your experiences with your partner over the last two weeks. How often did you and your partner argue with each other?"; Braiker \& Kelley, 1979). Possible responses were on a 7-point Likert scale (1 = not very often, 7 = very often), with higher scores indicating higher conflict at the onset of the pandemic $(M=2.41, S D=1.71)$ and in the follow-up surveys $(M=2.20, S D=1.54)$.

\section{Results}

\section{COVID-Related Stressors and Relationship Quality at the Onset of the Pandemic}

The data and syntax for all analyses reported for this paper can be found on the OSF. Correlations between all variables are presented in Table 1.

Table 1

Correlations Among Focal Variables 


$\begin{array}{llllllll}1 & 2 & 3 & 4 & 5 & 6 & 7 & 8\end{array}$

\section{Between-Person}

\section{Loneliness}

2. Financial Strain $.18 * * * \quad-$

3. Stress $.53 * * * \quad .15 * * *$

4. PPR - $-20 * * * \quad .01 \quad-.11 * * \quad-$

5. Relationship Sat. $\quad \begin{array}{llllll}-.23 * * * & -.05 & -.15 * * * & .63 * * * & \text { - }\end{array}$

6. Commitment $\quad-.13 * * * \quad-.01 \quad-.03 \quad .35 * * * \quad .59 * * * \quad-$

$\begin{array}{lllllll}\text { 7. Conflict } & .23 * * * & .10 * * & .25 * * * & -.39 * * * & -.38 * * * & -.20 * * *\end{array}$

8. Relationship Len. $\quad-.15 * * * \quad-.01 \quad-.15 * * * \quad-.21 * * * \quad-.12 * * * \quad-.04 \quad-.03$

\section{Within-Person}

\section{Loneliness}

2. Financial Strain $\quad .05^{* *} \quad-$

3. Stress $.30 * * * \quad .08 * * *$

4. PPR - $-.14 * * * \quad .04 \quad-.04 * \quad-$

5. Relationship Sat. $\quad-.16^{* * *} \quad .00 \quad-.09 * * * \quad .46 * * * \quad-$

6. Commitment $\quad-.04 * * \quad .00 \quad-.04 * \quad .22 * * * \quad .40 * * * \quad-$

$\begin{array}{llllllll}\text { 7. Conflict } & .12 * * * & .01 & .11 * * * & -.29 * * * & -.32 * * * & -.15 * * * & -\end{array}$

$\begin{array}{lllllllll}\text { 8. Relationship Len } & -.01 & .01 & -.01 & -.01 & -.03 & -.00 & .00\end{array}$

Note: $* * * p<.001, * * p<.01, * p<.05$. Baseline (Wave 1) reports of specific variables (perceived partner responsiveness and relationship length) were included in correlations. All other variables were assessed at all waves (bi-weekly) and aggregated across waves. PPR = 
Perceived partner responsiveness; Relationship Sat. = Relationship satisfaction. Relationship

Len. = Relationship length

Using multiple regression, we found that when people reported more loneliness or financial strain at onset of the pandemic, they reported feeling less satisfied and committed to their relationship and reported more conflict with their partner, and when people reported more stress, they reported less relationship satisfaction and more conflict, but reports of stress were not associated with people's reports of commitment. ${ }^{4}$

Table 2

Main Effects Models with Reports of COVID-related Stressors (Loneliness, Financial Strain, and Stress) Predicting Relationship Quality and Conflict at Wave 1

\begin{tabular}{lcccccccccc}
\hline & \multicolumn{3}{c}{ Relationship Satisfaction } & \multicolumn{3}{c}{ Commitment } & \multicolumn{3}{c}{ Conflict } \\
\cline { 2 - 10 } & $\boldsymbol{\beta}(\boldsymbol{S E})$ & $\boldsymbol{p}$-value & $\boldsymbol{C I}$ & $\boldsymbol{\beta}(\mathrm{SE})$ & $\boldsymbol{p}$-value & $\boldsymbol{C I}$ & $\boldsymbol{\beta}(\boldsymbol{S E})$ & $\boldsymbol{p}$-value & $\boldsymbol{C I}$ \\
\hline Loneliness & $-.20(.02)$ & .000 & $-.24,-.17$ & $-.12(.02)$ & .000 & $-.15,-.09$ & $.29(.02)$ & .000 & $.24, .33$ \\
FS & $-.08(.02)$ & .000 & $-.12,-.04$ & $-.03(.02)$ & .036 & $-.07,-.00$ & $.13(.03)$ & .000 & $.08, .18$ \\
Stress & $-.13(.02)$ & .000 & $-.17,-.09$ & $-.01(.02)$ & .548 & $-.05, .02$ & $.34(.03)$ & .000 & $.29, .39$
\end{tabular}

Note: $\mathrm{FS}=$ Financial Strain .

\section{Moderating Role of Perceived Partner Responsiveness at the Onset of the Pandemic}

In separate models, we assessed whether perceived partner responsiveness moderated associations by each COVID-related stressor by adding perceived partner responsiveness and the interaction between perceived partner responsiveness and COVID-related stressors (either loneliness, financial strain, or stress) to the model. When an interaction was significant, we tested

\footnotetext{
${ }^{4}$ In addition to assessing loneliness, stress, and financial strain, we also assessed worry about getting COVID, which was positively associated with relationship quality. We also assessed the effects of COVID-related stressors on relationship connection and found similar effects to those reported in the paper. Please see the Supplemental Materials for more information about the effects of worry and relationship connection.
} 
simple effects at high (one standard deviation above) and low (one standard deviation below) levels of perceived partner responsiveness (Aiken, West, \& Reno, 1991). Overall, the results suggest that perceived partner responsiveness mitigated the associations between COVID-related stressors and relationship quality (see Table 3). Specifically, for people who perceived their partner as low in responsiveness, COVID-related stress, financial strain, and loneliness were associated with significantly lower relationship satisfaction and more conflict in their relationship. However, for people who perceived their partner as highly responsive the association between COVID-related stressors and relationship quality were attenuated or completely buffered (see Table 3 for all values). There were three exceptions to this pattern of results: effects between loneliness and financial strain on commitment were not significantly moderated by perceived partner responsiveness; and the association between stress and commitment, although significantly moderated by perceived partner responsiveness, the simple slopes at both high and low levels of perceived partner responsiveness were not significant. As such, people who perceived their partners as highly responsive were buffered against the effect of COVID-related stress on their relationship satisfaction and experiences of conflict, but they were not significantly buffered against lower commitment.

Table 3

Interaction Models with Reports of COVID-related Stressors (Loneliness, Financial Strain (FS), and Stress) and Perceived Partner Responsiveness (PPR) Predicting Relationship Quality and Conflict at Wave 1

\begin{tabular}{llllllllll}
\multicolumn{2}{l}{ Relationship Satisfaction } & \multicolumn{3}{c}{ Commitment } & \multicolumn{3}{c}{ Conflict } \\
\hline$\beta(S E)$ & $p$-val. & $C I$ & $\beta(S E)$ & $p$-val. & $C I$ & $\beta(S E)$ & $p$-val. & $C I$
\end{tabular}

\section{Loneliness}




$\begin{array}{lccccccccc}\text { Loneliness } & -.13(.02) & .000 & -.16,-.10 & -.07(.02) & .000 & -.10,-.05 & .22(.02) & .000 & .18, .27 \\ \text { PPR } & .68(.02) & .000 & .65, .71 & .40(.02) & .000 & .37, .43 & -.58(.02) & .000 & -.62,-.53 \\ \text { Loneliness x PPR } & .06(.01) & .000 & .04, .09 & .01(.01) & .239 & -.01, .04 & -.05(.02) & .005 & -.09,-.02 \\ & & & & & & & & & \\ \text { Low PPR } & -.20(.02) & .000 & -.24,-.16 & - & - & - & .28(.03) & .000 & .22, .34 \\ \text { High PPR } & -.05(.02) & .009 & -.09,-.01 & - & - & - & .16(.03) & .000 & .10, .22\end{array}$

\section{Financial Strain (FS)}

$\begin{array}{lccccccccc}\text { FS } & -.05(.02) & .001 & -.08,-.02 & -.02(.02) & .284 & -.05, .01 & .11(.02) & .000 & .06, .15 \\ \text { PPR } & .70(.02) & .000 & .67, .73 & .40(.02) & .000 & .37, .43 & -.60(.02) & .000 & -.64,-.55 \\ \text { FS x PPR } & .03(.01) & .035 & .00, .05 & .02(.01) & .086 & -.00, .05 & -.07(.02) & .000 & -.10,-.03 \\ & & & & & & & & & \\ \text { Low PPR } & -.08(.02) & .000 & -.12,-.04 & - & - & - & .18(.03) & .000 & .12, .24 \\ \text { High PPR } & -.02(.02) & .395 & -.06, .02 & - & - & - & .03(.03) & .366 & -.03, .09\end{array}$

\section{Stress}

$\begin{array}{lccccccccc}\text { Stress } & -.10(.02) & .000 & -.13,-.07 & .01(.02) & .525 & -.02, .04 & .32(.02) & .000 & .27, .36 \\ \text { PPR } & .69(.02) & .000 & .66, .72 & .41(.02) & .000 & .38, .44 & -.59(.02) & .000 & -.63,-.54 \\ \text { Stress x PPR } & .05(.01) & .000 & .03, .08 & -.02(.01) & .070 & -.05, .00 & -.08(.02) & .000 & -.12,-.04 \\ & & & & & & & & \\ \text { Low PPR } & -.17(.02) & .000 & -.21,-.12 & .04(.02) & .092 & -.01, .09 & .41(.03) & .000 & .34, .48 \\ \text { High PPR } & -.04(.02) & .072 & -.08, .00 & -.02(.02) & .413 & -.06, .03 & .22(.03) & .000 & .16, .28\end{array}$

Note: $p$-val. $=p$-value. The effects for the interaction model are presented for each predictor, followed by the simple slopes presented at high PPR (one standard deviation above) and low PPR (one standard deviation below) levels of perceived partner responsiveness (PPR).

\section{Effects of COVID-related Stressors and Perceived Partner Responsiveness Overtime}

As stressors can change over the course of the pandemic, in the next set of analyses we look at within-person changes in COVID-related stressors and relationship outcomes to see if, at 
times when people report more stressors than typical, they report lower relationship quality and if these associations are buffered when partners are perceived to be highly responsive. To test these longitudinal effects, we used multilevel modelling using mixed models in SPSS (version 27.0) with timepoints nested within-person. We included random intercepts in the longitudinal models and tested for random slopes. For our longitudinal analyses, we tested models analyzing both the within-person (i.e., change within people over the three months) and between-person (i.e., difference between people over the three months) effect by entering both the person-mean centered and aggregated predictors in the models. We tested our key predictions about the detriments of COVID-related stressors on relationship quality, and the buffering effects of perceived partner responsiveness in separate models. That is, we first tested for a significant association between COVID-related stressors (each assessed separately) and our three key outcomes: 1) relationship satisfaction, 2) commitment, and 3) conflict. Then, in a follow-up model, we included all possible between and within-person interactions between a COVIDrelated stressor (either loneliness, financial strain, or stress) and perceived partner responsiveness at baseline (grand-mean centered) to test whether perceived partner responsiveness buffers the effects of COVID-related stressors on relationships. When associations were significantly moderated by perceptions of a partner's responsiveness, we tested simple slopes at low (-1SD) and high (+1SD) levels of perceived partner responsiveness.

When people reported more loneliness than their average, they reported lower relationship satisfaction and commitment and more conflict with their partner. Similarly, when stress was higher than average, people reported lower satisfaction, less commitment, and more conflict with their partner (see Table 4). However, largely, these effects were more pronounced among people who perceived their partners as low in responsiveness and were attenuated among 
those with highly responsive partners (see Table 5). When people perceived their partners as low in responsiveness and reported higher than average loneliness and stress, they reported poorer relationship satisfaction and more conflict with their partner. But when people perceived their partners as high in responsiveness, these effects were attenuated or reduced to non-significant. Within-person changes in financial strain were not associated with relationship quality or conflict, and the association between COVID-related stressors and commitment were not moderated by perceptions of a partner's responsiveness. Importantly, although not presented intext, the buffering effect of PPR assessed at baseline is largely consistent with the buffering effects over time when PPR was assessed as a time-varying moderator. That is, within-person changes in PPR over the course of the pandemic also buffered against associations between higher stressors and lower relationship quality (see Supplement 5 in the Supplemental Materials). Also, although not reported, the models we examined included between-person associations, and these were also consistent with the within-person effects in most cases (for more details see Supplement 3).

Table 4

Main Effects Models with Longitudinal Reports of COVID-related Stressors (Loneliness, Financial Strain, and Stress) Predicting Relationship Quality and Conflict

\begin{tabular}{lcccccccccc}
\hline & \multicolumn{3}{c}{ Relationship Satisfaction } & \multicolumn{3}{c}{ Commitment } & \multicolumn{3}{c}{ Conflict } \\
\cline { 2 - 10 } & $\boldsymbol{\beta}(\boldsymbol{S E})$ & $\boldsymbol{p}$-value & $\boldsymbol{C I}$ & $\boldsymbol{\beta}(\mathrm{SE})$ & $\boldsymbol{p}$-value & $\boldsymbol{C I}$ & $\boldsymbol{\beta}(\boldsymbol{S E})$ & $\boldsymbol{p}$-value & $\boldsymbol{C I}$ \\
\hline Loneliness & $-.18(.02)$ & .000 & $-.23,-.14$ & $-.04(.02)$ & .024 & $-.07,-.00$ & $.20(.03)$ & .000 & $.14, .26$ \\
FS & $-.00(.03)$ & .949 & $-.04, .05$ & $-.00(.02)$ & .870 & $-.05, .03$ & $.01(.03)$ & .686 & $-.05, .08$ \\
Stress & $-.09(.02)$ & .000 & $-.14,-.05$ & $-.04(.02)$ & .018 & $-.07,-.01$ & $.19(.03)$ & .000 & $.13, .25$
\end{tabular}

Note: $\mathrm{FS}=$ Financial Strain . 
Table 5

Interaction Models with Longitudinal Reports of COVID-related Stressors (Loneliness,

Financial Strain (FS), and Stress) and Perceived Partner Responsiveness (PPR) Predicting

Relationship Quality and Conflict

\section{Relationship Satisfaction}

Commitment

Conflict

$\begin{array}{lllllllll}\beta(S E) & p \text {-val. } & C I & \beta(S E) & p \text {-val. } & C I & \beta(S E) & p \text {-val. } & C I\end{array}$

\section{Loneliness}

$\begin{array}{lccccccccc}\text { Loneliness } & -.18(.02) & .000 & -.22,-.13 & -.04(.02) & .016 & -.07,-.01 & .20(.03) & .000 & .13, .26 \\ \text { PPR } & .60(03) & .000 & .55, .65 & .31(.03) & .000 & .26, .36 & -.40(.03) & .000 & -.46,-.33 \\ \text { Loneliness x PPR } & .08(.02) & .000 & .04, .12 & -.00(.01) & .917 & -.03, .03 & -.07(.03) & .008 & -.12,-.02 \\ & & & & & & & & & \\ \text { Low PPR } & -.27(.03) & .000 & -.33,-.21 & - & - & - & .28(.04) & .000 & .19, .36 \\ \text { High PPR } & -.09(.03) & .007 & -.15,-.02 & - & - & - & .11(.05) & .014 & .02, .20\end{array}$

Financial Strain (FS)

$\begin{array}{llllllllll}\text { FS } & .01(.02) & .820 & -.04, .05 & .00(.02) & .981 & -.04, .04 & .00(.03) & .900 & -.06, .07 \\ \text { PPR } & .63(.03) & .000 & .58, .68 & .32(.02) & .000 & .27, .36 & -.42(.03) & .000 & -.49,-.36 \\ & & & & & & & & & \\ \text { FS x PPR } & .02(.02) & .244 & -.02, .06 & .02(.02) & .194 & -.01, .05 & -.03(.03) & .322 & -.08, .03\end{array}$

Low PPR

High PPR

Stress

$\begin{array}{lccccccccc}\text { Stress } & -.10(.02) & .000 & -.14,-.05 & -.03(.02) & .028 & -.07,-.00 & .18(.03) & .000 & .12, .25 \\ \text { PPR } & .61(.02) & .000 & .57, .66 & .32(.02) & .000 & .27, .37 & -.40(.03) & .000 & -.46,-.34 \\ \text { Stress x PPR } & .04(.02) & .020 & .01, .08 & .01(.01) & .498 & -.02, .03 & -.05(.03) & .053 & -.11, .00 \\ & & & & & & & & & \\ \text { Low PPR } & -.15(.03) & .000 & -.21,-.09 & - & - & - & .24(.04) & .000 & .16, .33\end{array}$


$\begin{array}{lllllllllll}\text { High PPR } & -.05(.03) & .153 & -.11, .02 & - & - & - & .12(.04) & .006 & .04, .21\end{array}$

Note: $* * * p<.001, * * p<.01, * p<.05$. The effects for the interaction model are presented for each predictor, followed by the simple slopes presented at high PPR (one standard deviation above) and low PPR (one standard deviation below) levels of perceived partner responsiveness (PPR).

\section{Providing Evidence for Generalizability}

Given that relationship satisfaction tends to decline on average with increasing relationship duration (Karney \& Bradbury, 1995; Kurdek, 1999) and that coping with COVIDrelated stressors may be different for couples cohabiting versus not living together, we conducted auxiliary analyses to test whether any of the effects are accounted for by relationship factors (such as relationship duration and cohabitation status) or social distancing restrictions. Largely, the findings reported above remained significant after accounting for how long couples had been together or whether they lived together, though in some cases differences emerged. For example, when accounting for relationship length and cohabitation, the relationship between financial strain and relationship quality differed, and perceived partner responsiveness no longer emerged. In contrast, two moderations were gained when cohabitation status was controlled for. That is, the association between loneliness and commitment, and stress and commitment, were now moderated by perceptions of a partner's responsiveness (for details see the Supplemental Materials).

Also, given that our data consisted of people from 57 different countries and that social distancing guidelines and regulations differed across country and region, we decided a priori to explore whether the local-level social distancing regulations influenced the association between COVID-related stressors and relationship quality as well as the buffering effects of perceived 
partner responsiveness. To do so, we examined the effects of interest while controlling for social distancing regulations. More specifically, we examined three items that assessed local or national policies specific to social distancing (i.e., "social distancing has been encouraged," "social distancing has been ordered," and "social distancing is being enforced by the police", coded as 1, 2, or 3, respectively). Overall, at baseline, in $20.6 \%$ of cases social distancing had been encouraged, in $32.9 \%$ ordered, in $42.3 \%$ enforced, and in $4.1 \%$ of cases no social distancing policies had been reported. All the effects reported above remained significant after accounting for social distancing policies, both at baseline and overtime (see Supplement 1 for more information).

\section{Discussion}

In the current study of people in relationships across 57 countries, we found that people who reported greater loneliness, financial strain, and stress brought on by the COVID-19 pandemic reported lower relationship satisfaction, less commitment, and more conflict in their relationship at the onset of the pandemic. Changes in COVID-related stressors over the course of the pandemic were also associated with lower satisfaction and more conflict when people experienced higher loneliness and stress. However, people who perceived their partner as highly responsive were in some instances buffered against these associations between COVID-related stressors and lower relationship quality, especially at the onset of the pandemic (though effects were less robust over time). These findings suggest that having a partner who is seen as responsive to one's needs (e.g., high in perceived partner responsiveness) may be an important factor in mitigating the spillover of external stressors, like those introduced by COVID-19, into a romantic relationship, and can help couples experiencing stress maintain relationship quality, especially relationship satisfaction and lower levels of conflict, overtime. 


\section{COVID-Related Stressors and Relationship Quality}

The current findings corroborate prior work demonstrating the powerful role that social disconnection (e.g., Flora \& Segrin, 2000; Segrin et al., 2003), financial strain (Bodenmann, 1997; Conger et al., 1999; Karney et al., 2005; Story \& Bradbury; 2004), and external stressors (e.g., Randall \& Bodenmann, 2009) play in shaping relationship processes. Indeed, the results provide compelling evidence for the association between COVID-related stressors and relationship quality. The findings are also in line with the stress spillover phenomena (e.g., Bolger et al., 1989; Buck \& Neff, 2012; Neff \& Karney, 2004; Randall \& Bodenmann, 2009), which posits that stressors originating in domains external to the relationship, such as the stressors introduced by the COVID-19 pandemic, predict decreases in relational satisfaction and increases in maladaptive relationship behaviors, such as conflict. However, some external stressors were more robust and reliable predictors of relationship quality and conflict over the course of the pandemic. More specifically, at the onset of the pandemic, loneliness, financial strain, and stress were all associated with lower levels of relationship satisfaction and commitment and greater conflict (albeit with weaker effects for financial strain), but when we examined the effects overtime we found that within-person changes in financial strain were not associated with relationship satisfaction, commitment, or conflict, whereas within-person changes in loneliness and stress were both associated with relationship satisfaction and conflict (though not with commitment). These findings are in line with research recent research that found that perceived stress, but not economic pressure or pandemic concerns, were associated with increases in relationship instability (Ogan, Monk, Kanter, \& Proulx, 2021).

\section{Buffering Effect of Perceived Partner Responsiveness}


Although past research on perceived partner responsiveness has highlighted the benefits to having a partner who is perceived to be responsive, to date, there is a dearth of research examining the role of responsiveness in buffering naturally occurring stressful events, or in moderating the effects of people's reactions to external stressors. In fact, to our knowledge, no past work has assessed the role of perceived partner responsiveness as a protective factor against external stressors such as loneliness and financial strain on relationship quality. The COVID-19 pandemic and the ensuing stay-at-home orders and economic effects provide a unique opportunity to understand protective factors for stress spillover into relationships. Yet, while COVID-19 is a unique experience in recent history, people often face stress brought on by a variety of external factors, such as natural disasters, economic recessions, and job-related stress/loss. This work suggests that having a responsive partner might be especially helpful for individuals facing loneliness or stress, but the results are generally weaker for financial strain. There is a dearth of research examining the effects of responsiveness on financial stressors though it is possible that responsiveness is less effective at alleviating the strain from financial stress as financial concerns may be less addressable through a partner's support. That is, when a partner feels lonely or stressed, a responsive partner could provide support through either spending time with their partner (to relieve loneliness) or through talking about the stressors (i.e., understanding, listening to, and being supportive of the stress), though a partner's responsiveness may not be as effective at alleviating financial concerns because these stressors may persist despite a partner's responsiveness (e.g., if an individual loses their job, a partner's support and responsiveness might not be enough to help relieve the financial strain of losing a job). This would seem to merit attention in future research, in as much as financial strain, as a predictor, is more distinct from perceived partner responsiveness and relationship quality than loneliness and 
stress are, and thus, this might suggest that the stress buffering effects are limited to certain contexts. Thus, future research could examine the generalizability of perceived partner responsiveness in buffering the association between other external stressors and relationship quality.

We want to note that perceived partner responsiveness had a small, negative correlation with the extent to which people were experiencing COVID-related stressors. That is, people who reported fewer or less severe stressors perceived their partners as more responsive. On the one hand, this could suggest that it might be easier to perceive a partner as responsive when external stressors are lower. Alternatively, it is possible that people who report high levels of perceived partner responsiveness might perceive and appraise external stressors to be less stressful whereas people who do not have the support of a responsive partner might catastrophize stressors. This line of reasoning is consistent with the vulnerability-stress-adaption (VSA) model (Karney \& Bradbury, 1995) and the stress buffering model (Cohen \& Wills, 1985; Slatcher \& Selcuk, 2017) which suggests that protective factors - like a supportive relationship - can buffer the adverse effects of stress. Indeed, a supportive environment and partner might help dampen the effects of stress, allowing couples to better cope with stressors encountered (e.g., Mueser \& Glynn, 1990) and in turn, maintain satisfying relationships. Importantly, the correlations were small, which suggests that even people who perceived their partner as responsive were still likely experiencing external stressors in the wake of COVID-19.

Our results suggest that while perceived partner responsiveness moderated many of the associations between COVID-related stressors and relationship satisfaction and conflict, the effects were either weak or null for commitment - that is, even if satisfaction is lower and conflict is higher, people may remain committed to their relationship when faced with external 
stressors. It is possible that in times of stress, people might experience more conflict and less satisfaction, but remain committed to their relationships given their investment in the relationship during a time of economic uncertainty and fewer other social ties and alternatives to the relationship (Rusbult, Martz, \& Agnew, 1998). Although not tested in the current study, one explanation for these effects is that commitment may be less impacted by external stressors to the relationship during a pandemic, regardless of a partner's responsiveness, in part because social distancing measures that have been put in place to curb the spread of COVID-19 (Koo et al., 2020; Lewnard \& Lo, 2020) may have restricted individual's opportunities to seek out and meet new potential partners. As such, and in line with Investment Model (Rusbult et al., 1998), one's alternatives to leaving their relationship may have been impacted during the pandemic, which sustained commitment even in times of lower satisfaction (or when a partner is seen as less responsive). Additionally, it is important to note that the buffering effects of perceived partner responsiveness emerged more consistently at the onset of the pandemic, with $7 / 9$ of the instances examined presenting evidence in support of the buffering effect. Whereas when we examined these effects over time, the protective armor of perceived partner responsiveness diminished, with only $3 / 9$ of the instances examined presenting evidence in support of the protective role.

\section{Implications, Limitations, and Future Directions}

Although this research provides initial insights into the link between COVID-related stressors and relationship quality and the buffering role of perceived partner responsiveness, the current analyses rely on the reports of individuals who completed the survey shortly after stay-athome orders were mandated and we are unable to account for people's relationship quality prior to the pandemic. Therefore, it remains unclear how people's relationship quality prior to the 
pandemic influenced their reports of stressors and responsiveness over the course of the pandemic. It is likely people who were highly satisfied in their relationships before COVID-19 pandemic would also report higher relationship quality during and post-COVID, as well as perceive their partners as more responsive (Williamson, 2021). Although we cannot test this directly, we did examine whether people who participated in the study at baseline differed from those who participated in the study over time (see the Supplemental Materials) and we found that in some cases, participants who completed the baseline survey only differed from those who completed the surveys over the course of the pandemic. More specifically, in comparison to participants who remained in the study over time, participants who completed the baseline survey only, on average, reported greater loneliness, financial strain, and conflict, and they were less committed to their partner and perceived their partner to be less responsive at the onset of the pandemic. As such, one limitation of this study is that participants who were experiencing high levels of COVID-related stressors and lower levels of relationship quality did not remain in the study and it is possible that the effects would have differed, or have been more robust, among those who were more impacted by COVID-19 and who had poorer quality relationships. In addition, the current research relies on reports of individuals and their perceptions of their partner's responsiveness, whether these perceptions reflect actual responsive behaviors from a partner (and behaviors are perceived as responsive) is not clear from this study. Future work might aim to understand how a partner's enacted responsiveness shapes perceptions of responsiveness, and importantly, what it is that responsive partners are actually doing to help their partners cope with COVID-related stressors. Finally, despite efforts to include respondents from diverse backgrounds (e.g., recruiting participants from 57 different countries and who speak 11 different languages) and to include non-WEIRD cultures, the current research resulted 
in responses from participants who were largely from Western cultures. This is potentially a limitation and future research should seek to recruit a more diverse sample that is adequately designed to assess whether and under what circumstances culture shapes COVID-related effects on relationships.

\section{Conclusion}

Stressful events are inevitable, can be difficult to manage, and often take a toll on people's close relationships. In the current study, we demonstrate that COVID-related stressors are negatively associated with evaluations of relationship quality and positively associated with people's reports of conflict with their partner. However, we demonstrate that perceived partner responsiveness - the extent to which people believe that their partners understand, validate, and care for them - in relationships may confer protective "armor" against spillover of these stressors into their relationship and may help people sustain high quality relationships in the face of stress. 


\section{References}

Aiken, L. S., West, S. G., \& Reno, R. R. (1991). Multiple regression: Testing and interpreting interactions. Sage.

Baumeister, R. F., \& Leary, M. R. (1995). The need to belong: Desire for interpersonal attachments as a fundamental human motivation. Psychological Bulletin, 117, 497-529.

Bennett, D. A. (2001). How can I deal with missing data in my study?. Australian and New Zealand Journal of Public Health, 25, 464-469.

Berinsky, A. J., Margolis, M. F., \& Sances, M. W. (2014). Separating the shirkers from the workers? Making sure respondents pay attention on self-administered surveys. American Journal of Political Science, 58, 739-753.

Birnbaum, G. E., Reis, H. T., Mizrahi, M., Kanat-Maymon, Y., Sass, O., \& Granovski-Milner, C. (2016). Intimately connected: The importance of partner responsiveness for experiencing sexual desire. Journal of Personality and Social Psychology, 111, 530-546.

Bodenmann, G. (1997). The influence of stress and coping on close relationships: A two year longitudinal study. Swiss Journal of Psychology, 56, 156-164.

Bolger, N., Davis, A., \& Rafaeli, E. (2003). Diary methods: Capturing life as it is lived. Annual Review of Psychology, 54, 579-616.

Bolger, N., DeLongis, A., Kessler, R. C., \& Wethington, E. (1989). The contagion of stress across multiple roles. Journal of Marriage and the Family, 51, 175-183.

Buck, A. A. \& Neff, L. A. (2012). Stress spillover in early marriage: The role of self-regulatory depletion. Journal of Family Psychology, 26, 698-708. 
Braiker, H. B., \& Kelley, H. H. (1979). Conflict in the development of close relationships. In R. L. Burgess \& T. L. Huston (Eds.), Social exchange in developing relationships (pp. 135168). New York, NY: Academic Press.

Brooks, S. K., Webster, R. K., Smith, L. E., Woodland, L., Wessely, S., Greenberg, N., et al. (2020). The psychological impact of quarantine and how to reduce it: Rapid review of the evidence. Lancet, 395, 912-920.

Caprariello, P. A., \& Reis, H. T. (2011). Perceived partner responsiveness minimizes defensive reactions to failure. Social Psychological and Personality Science, 2, 365-372.

Carlson, D. L., Petts, R., \& Pepin, J. R. (2020). US couples' division of housework and childcare during COVID-19 pandemic. Retrieved from https://osf.io/preprints/socarxiv/jy8fn.

Clark, M. S., \& Lemay, J. E. P. (2010). Close relationships. In S. T. Fiske, D. T. Gilbert, \& G. Lindzey (Eds.), Handbook of social psychology (Vol. 2, 5th ed., pp. 898-940). Hoboken, NJ: John Wiley \& Sons.

Cohan, C. L., \& Cole, S. W. (2002). Life course transitions and natural disaster: Marriage, birth, and divorce following Hurricane Hugo. Journal of Family Psychology, 16, 14 -25.

Cohen, S., \& Wills, T. A. (1985). Stress, social support, and the buffering hypothesis. Psychological Bulletin, 98, 310-357.

Colina, S., Marrone, N., Ingram, M., \& Sanchez, D. (2017). Translation quality assessment in health research: A functionalist alternative to back-translation. Evaluation \& the Health Professions, 40, 267-293.

Collins, N. L., \& Feeney, B. C. (2000). A safe haven: An attachment theory perspective on support seeking and caregiving in intimate relationships. Journal of Personality and Social Psychology, 78, 1053-1073. 
Conger, R. D., Rueter, M. A., \& Elder, G. H. (1999). Couple resilience to economic pressure. Journal of Personality and Social Psychology, 76, 54-71.

Congressional Research Service. (2020). Global Economic Effects of COVID-19. Retrieved from https://fas.org/sgp/crs/row/R46270.pdf.

Crasta, D., Maniaci, M. R., Rogge, R. D., \& Reis, H. T. (under review). Clarifying the measurement of Perceived Partner Responsiveness: The Perceived Responsiveness and Insensitivity Scale.

Crasta, D., Rogge, R. D., Maniaci, M. R., \& Reis, H. T. (2021). Toward an optimized measure of perceived partner responsiveness: Development and validation of the Perceived Responsiveness and Insensitivity Scale. Psychological Assessment. Advance online publication.

Erdfelder, E., Faul, F., \& Buchner, A. (1996). GPOWER: a general power analysis program. Behavior Research Methods, Instruments, \& Computers, 28, 1-11.

Ervasti, H. \& Venetoklis, T. (2010). Unemployment and subjective well-being: an empirical test of deprivation theory, incentive paradigm and financial strain approach. Acta Sociologica $53,119-138$.

Faria e Castro, M. (2020). Fiscal policy during a pandemic. Federal Reserve Bank of St. Loius. Retrieved from https://s3.amazonaws.com/real.stlouisfed.org/wp/2020/2020-006.pdf Faul, F., Erdfelder, E., Buchner, A., \& Lang, A. G. (2009). Statistical power analyses using G* Power 3.1: tests for correlation and regression analyses. Behavior Research Methods, 41, $1149-1160$. 
Fletcher, G. J. O., Simpson, J. A., \& Thomas, G. (2000). The measurement of perceived relationship quality components: A confirmatory factor analytic approach. Personality and Social Psychology Bulletin, 26, 340-354.

Flora, J., \& Segrin, C. (2000). Relationship development in dating couples: Implications for relational and personal well-being. Journal of Social and Personal Relationships, 17, $811-825$.

Gable, S. L., Reis, H. T., Impett, E. A., \& Asher, E. R. (2004). What do you do when things go right? The intrapersonal and interpersonal benefits of sharing positive events. Journal of Personality and Social Psychology, 87, 228-245.

Gangopadhyaya, A., \& Garrett, B. (2020). Unemployment, Health Insurance, and the COVID19 Recession. Urban Institute.

Gritz, E. R., Wellisch, D. K., Siau, J., \& Wang, H. (1990). Long-term effects of testicular cancer on marital relationships. Psychosomatics, 31, 301-312.

Hawkley, L. C., Browne, M. W., \& Cacioppo, J. T. (2005). How can I connect with thee? Let me count the ways. Psychological Science, 16, 798-804.

Holt-Lunstad, J., Smith, T. B., \& Layton, J. B. (2010). Social relationships and mortality risk: A meta-analytic review. PLoS Medicine, 7, 1-20.

Hughes, M. E., Waite, L. J., Hawkley, L. C., \& Cacioppo, J. T. (2004). A short scale for measuring loneliness in large surveys: Results from two population-based studies. Research on Aging, 26, 655-672.

International Labor Organization (2020). ILO Monitor: COVID-19 and the world of work. Second edition. Retrieved from 
https://www.ilo.org/wcmsp5/groups/public/@dgreports/@dcomm/documents/briefingnot e/wcms_740877.pdf.

Johnson, D., \& Mullins, L. (1989). Religiosity and loneliness among the elderly. Journal of Applied Gerontology, 8, 110-131.

Karney, B. R., and Bradbury, T. N. (1995). The longitudinal course of marital quality and stability: A review of theory, methods, and research. Psychological Bulletin, 118, 3-34.

Karney, B. R., Story, L. B., \& Bradbury, T. N. (2005). Marriages in context: Interactions between chronic and acute stress among newlyweds. In T. A. Revenson, K. Kayser, \& G. Bodenmann (Eds.). Couples coping with stress: Emerging perspectives on dyadic coping (pp. 13-32). Washington, DC: American Psychological Association Press.

Karney, B. R., \& Neff, L. A. (2013). Couples and stress: How demands outside a relationship affect intimacy within the relationship. In J. A. Simpson \& L. Campbell (Eds.). The Oxford Handbook of Close Relationships (pp. 664-684). New York, NY: Oxford University Press.

Kerr, M. L., Rasmussen, H. F., Fanning, K. A., \& Braaten, S. M. (2021). Parenting during COVID-19: A study of parents' experiences across gender and income levels. Family Relations. Advance online publication.

King, A. (2020). Official unemployment numbers don't show the true crisis for workers affected by coronavirus. The Conversation. Retrieved from https://theconversation.com/officialunemployment-numbers-dont-show-the-true-crisis-for-workers-affected-by-coronavirus$\underline{136110 .}$

Kochhar, R. (2020). Unemployment rose higher in three months of COVID-19 than it did in the years of the Great Recession. Pew Research Center. Retrieved from 
https://www.pewresearch.org/fact-tank/2020/06/11/unemployment-rose-higher-in-threemonths-of-covid-19-than-it-did-in-two-years-of-the-great-recession/.

Koo, J. R., Cook, A. R., \& Park, M. (2020). Interventions to mitigate early spread of COVID-19 in Singapore: a modelling study. Lancet Infectious Diseases. Advance online publication.

Kurdek, L. A. (1999). The nature and predictors of the trajectory of change in marital quality for husbands and wives over the first 10 years of marriage. Developmental Psychology, 35, 1283-1296.

Laurenceau, J. P., Barrett, L. F., \& Pietromonaco, P. R. (1998). Intimacy as an interpersonal process: The importance of self-disclosure, partner disclosure, and perceived partner responsiveness in interpersonal exchanges. Journal of Personality and Social Psychology, 74, 1238-1251.

Laurenceau, J. P., Barrett, L. F., \& Rovine, M. J. (2005). The interpersonal process model of intimacy in marriage: A daily-diary and multilevel modeling approach. Journal of Family Psychology, 19, 314-323.

Lehman, D. R., Lang, E. L., Wortman, C. B., \& Sorenson, S. B. (1989). Long-term effects of sudden bereavement: Marital and parent-child relationships and children's reactions. Journal of Family Psychology, 2, 344 -367.

Lewnard, J. A. \& Lo, N. C. (2020). Scientific and ethical basis for social-distancing interventions against COVID-19. Lancet Infectious Diseases. Advance online publication.

Löwe, B., Wahl, I., Rose, M., Spitzer, C., Glaesmer, H., Wingenfeld, K., ... \& Brähler, E. (2010). A 4-item measure of depression and anxiety: validation and standardization of the Patient Health Questionnaire-4 (PHQ-4) in the general population. Journal of Affective Disorders, 122, 86-95. 
Maisel, N. C., Gable, S. L., \& Strachman, A. (2008). Responsive behaviors in good times and in bad. Personal Relationships, 15, 317-338.

Manne, S., \& Badr, H. (2008). Intimacy and relationship processes in couples' psychosocial adaptation to cancer. Cancer, 112, 2541-2555.

Manne, S., Kashy, D. A., Zaider, T., Lee, D., Kim, I. Y., Heckman, C., . . Virtue, S. M. (2018). Interpersonal processes and intimacy among men with localized prostate cancer and their partners. Journal of Family Psychology, 32, 664-675.

Manne, S., Ostroff, J., Rini, C., Fox, K., Goldstein, L., \& Grana, G. (2004). The interpersonal process model of intimacy: The role of self-disclosure, partner disclosure, and partner responsiveness in interactions between breast cancer patients and their partners. Journal of Family Psychology, 18, 589-599.

Mikulincer, M., \& Shaver, P. R. (2007). Attachment in adulthood: Structure, dynamics, and change. New York, NY: Guilford Press

Mikulincer, M., \& Shaver, P. R. (2013). The role of attachment security in adolescent and adult close relationships. In J. A. Simpson \& L. Campbell (Eds.), Oxford handbook of close relationships (pp. 66-89). New York, NY: Oxford University Press.

Neff, L. A., \& Karney, B. R. (2004). How does context affect intimate relationships? Linking external stress and cognitive processes within marriage. Personality and Social Psychology Bulletin, 30, 134-148.

Neff, L. A., \& Karney, B. R. (2017). Acknowledging the elephant in the room: How stressful environmental contexts shape relationship dynamics. Current Opinions in Psychology, $13,107-110$. 
Neff, L. A., Gleason, M. E. J., Crockett, E. E., \& Ciftci, O. (2021). Blame the pandemic: Buffering the association between stress and relationship quality during the COVID-19 pandemic. Social Psychological and Personality Science. Advance online publication.

Ogan, M. A., Monk, J. K., Kanter, J. B., \& Proulx, C. M. (2021). Stress, dyadic coping, and relationship instability during the COVID-19 pandemic. Journal of Social and Personal Relationships. Advance online publication.

Oggins, J. (2004). Attrition biases in a study of Euro-American and African-American marriages. Psychological Reports, 94, 1051-1057.

Overall, N. C., Chang, V., Pietromonaco, P. R., Low, R., \& Henderson, A. (2021). Partners' attachment insecurity and stress predict poorer relationship functioning during COVID19 quarantines. Social Psychological and Personality Science. Advance online publication.

Pappas, S. (2020). How will people react to the new financial crisis? American Psychological Association. Retrieved from https://www.apa.org/news/apa/2020/04/financial-crisisCOVID-19.

Pearlin, L. I., Lieberman, M. A., Menaghan, E. G., \& Mullan, J. T. (1981). The stress process. Journal of Health and Social Behavior, 22, 337-356.

Pietromonaco, P. R., \& Beck, L. A. (2019). Adult attachment and physical health. Current Opinion in Psychology, 25, 115-120.

Pietromonaco, P. R., \& Collins, N. L. (2017). Interpersonal mechanisms linking close relationships to health. American Psychologist, 72, 531-542.

Randall, A. K. \& Bodenmann, G. (2009). The role of stress on close relationships and marital satisfaction. Clinical Psychology Review, 29, 105-115. 
Reis, H. T. (2012). Perceived partner responsiveness as an organizing theme for the study of relationships and well-being. In L. Campbell \& T. J. Loving (Eds.), Interdisciplinary research on close relationships: The case for integration (pp. 27-52). Washington, DC: American Psychological Association.

Reis, H. T., \& Clark, M. S. (2013). Responsiveness. In J. A. Simpson \& L. Campbell (Eds.), Oxford handbook of close relationships (pp. 400-423). New York, NY: Oxford University Press.

Reis, H. T., Clark, M. S., \& Holmes, J. G. (2004). Perceived partner responsiveness as an organizing construct in the study of intimacy and closeness. In D. J. Mashek \& A. P. Aron (Eds.), Handbook of closeness and intimacy (pp. 201-225). Mahwah, NJ: Erlbaum Publishers.

Reis, H. T., Lemay, E. P., \& Finkenauer, C. (2017). Toward understanding: The importance of feeling understood in relationships. Social and Personality Psychology Compass, 11, 122.

Reis, H. T., \& Shaver, P. (1998). Intimacy as an interpersonal process. In S. Duck, D. F. Hay, S. E. Hobfoll, W. Ickes, \& B. M. Montgomery (Eds.), Handbook of personal relationships: Theory, research, and interventions (pp. 367-389). Oxford, England: John Wiley \& Sons.

Repetti, R. L. (1989). Effects of daily workload on subsequent behavior during marital interaction: The roles of social withdrawal and spouse support. Journal of Personality and Social Psychology, 57, 651-659. 
Repetti, R., Wang, S., \& Saxbe, D. (2009). Bringing it all back home: How outside stressors shape families' everyday lives. Current Directions in Psychological Science, 18, 106111.

Rusbult, C. E., Martz, J. M., \& Agnew, C. R. (1998). The investment model scale: Measuring commitment level, satisfaction level, quality of alternatives, and investment size. Personal Relationships, 5, 357-387.

Okechukwu, C. A., El Ayadi, A. M., Tamers, S. L., Sabbath, E. L., \& Berkman, L. (2012). Household food insufficiency, financial strain, work-family spillover, and depressive symptoms in the working class: The Work, Family, and Health Network study. American Journal of Public Health, 102, 126-133.

Schulz, M. S., Cowan, P. A., Cowan, C. P., \& Brennan, R. T. (2004). Coming home upset: Gender, marital satisfaction, and the daily spillover of workday experience into couples interactions. Journal of Family Psychology, 18, 250-263.

Segal, N., \& Fraley, R. C. (2016). Broadening the investment model: An intensive longitudinal study on attachment and perceived partner responsiveness in commitment dynamics. Journal of Social and Personal Relationships, 33, 581-599.

Segrin, C., Powell, H., Givertz, M., \& Brackin, A. (2003). Depression, relational quality, and loneliness in dating relationships. Personal Relationships, 10, 25-36.

Selcuk, E., Zayas, V., \& Hazan, C. (2010). Beyond satisfaction: The role of attachment in marital functioning. Journal of Family Theory \& Review, 2, 258-279.

Simpson, J. A., Rholes, W. S., \& Nelligan, J. S. (1992). Support seeking and support giving within couples in an anxiety provoking situation: The role of attachment styles. Journal of Personality and Social Psychology, 62, 434-446. 
Slatcher R. B. (2010). Marital functioning and physical health: Implications for social and personality psychology. Social and Personality Psychology Compass, 4, 455-469.

Slatcher, R. B., \& Schoebi, D. (2017). Protective processes underlying the links between marital quality and physical health. Current Opinion in Psychology, 13, 148-152.

Slatcher, R. B., \& Selcuk, E. (2017). A social psychological perspective on the links between close relationships and health. Current Directions in Psychological Science, 26, 16-21.

Story, L. B., \& Bradbury, T. N. (2004). Understanding marriage and stress: Essential questions and challenges. Clinical Psychology Review, 23, 1139-1162.

Tesser, A., \& Beach, S. R. H. (1998). Life events, relationship quality, and depression: An investigation of judgment discontinuity in vivo. Journal of Personality and Social Psychology, 74, 36-52.

The World Bank. (2021). GDP per capita (current US\$). Retrieved from https://data.worldbank.org/indicator/NY.GDP.PCAP.CD?most_recent_value_desc=true

Tyupa, S. (2011). A theoretical framework for back-translation as a quality assessment tool. New Voices in Translation Studies, 7, 35-46.

United Nations. (2020). The Social Impact of COVID-19. Retrieved from https://www.un.org/development/desa/dspd/2020/04/social-impact-of-COVID-19/

Watson, D., \& Clark, L. A. (1999). The PANAS-X: Manual for the positive and negative affect schedule-expanded form.

Williamson, H. C. (2021). Early Effects of the COVID-19 Pandemic on Relationship Satisfaction and Attributions. Psychological Science. Advance online publication. 
Williamson, H. C, Karney, B. R., \& Bradbury, T. N. (2013). Financial strain and stressful events predict newlyweds' negative communication independent of relationship satisfaction. Journal of Family Psychology, 27, 65-75.

World Health Organization (WHO). (2020). Rolling Updates on Coronavirus Disease (COVID19). Retrieved from https://www.who.int/emergencies/diseases/novel-coronavirus2019/events-as-they-happen.

World Trade Organization. (2020). Trade set to plunge as COVID-19 pandemic upends global economy. Retrieved from https://www.wto.org/english/news_e/pres20_e/pr855_e.htm. 